\title{
Impacto de la Telemedicina en la calidad del Control de Tratamiento Anticoagulante Oral
}

\author{
Elena Nieto ${ }^{1}$, Ángela Roco ${ }^{2}$, Bárbara Brintrup ${ }^{3}$, Carlos Ramírez ${ }^{4}$, Priscilla Barrera ${ }^{4}$, René \\ Meneses $^{3}$, Jorge Carabantes ${ }^{5}$, Juan Ovalle ${ }^{6}$, Irma Matus de la Parra ${ }^{7}$, Omar Zagmutt ${ }^{8}$, Carlos \\ Gallardo $^{9}$, Vladimir Pizarro ${ }^{10}$, Juan Cayún ${ }^{11}$.
}

\footnotetext{
1. Médico especialidad Laboratorio Clínico, Policlínico de Tratamiento Anticoagulante CDT-Hospital San Juan de Dios Servicio de Salud Metropolitano Occidente.

2. Bioquímico, Coordinación de Unidades de Apoyo, Departamento Coordinación de la Red Asistencial, Servicio de Salud Metropolitano Occidente.

3. Médico, Hospital de Curacaví, Servicio de Salud Metropolitano Occidente.

4. Enfermero (a) Hospital de Curacaví, Servicio de Salud Metropolitano Occidente.

5. Médico especialidad Cardiología, Policlínico de Tratamiento Anticoagulante CDT-Hospital San Juan de Dios Servicio de Salud Metropolitano Occidente.

6. Ingeniero Departamento Informática Hospital Curacaví Servicio de Salud Metropolitano Occidente.

7. Ingeniero Departamento Informática Hospital San Juan de Dios Servicio de Salud Metropolitano Occidente.

8. Médico Departamento de Procesos Asistenciales, Servicio de Salud Metropolitano Occidente.

9. Médico Departamento Coordinación de la Red Asistencial, Servicio de Salud Metropolitano Occidente.

10. Odontólogo, Director Servicio de Salud Metropolitano Occidente.

11. Químico Farmacéutico, Laboratorio de Carcinogénesis Química y Farmacogenética, Centro de Investigaciones Farmacológicas y Toxicológicas, ICBM, Facultad de Medicina, Universidad de Chile.
}

Antecedentes: En el año 2014 se inició Telemedicina desde el Policlínico de Tratamiento Anticoagulante oral del Hospital San Juan de Dios y el Hospital de Curacaví, evitando así el traslado de pacientes a Santiago para el control con el médico especialista.

Métodos: Se utilizó licencia de video conferencia en el Hospital San Juan de Dios, dispositivo móvil, equipo de INR capilar y stock de Acenocumarol en el Hospital de Curacaví.

Resultados: En total se han realizado 2.174 consultas vía Telemedicina (junio 2014 a diciembre 2015). Esta estrategia ha sido bien evaluada por los pacientes. La mejora en la calidad del tratamiento ha sido evidente: 58,3\% de los pacientes del Hospital de Curacaví se encuentran en rango terapéutico, supe- rior al 50,8\% de los pacientes del Hospital San Juan de Dios $(\mathrm{p}<0,05)$. En cuanto al Tiempo en Rango Terapéutico (TTR) 50,6\% de los pacientes del Hospital de Curacaví se encuentran en rango versus 46,2\% de los pacientes del Hospital San Juan de Dios ( $\mathrm{p}<$ $0,05)$.

Conclusiones: La Telemedicina utilizada por equipos comprometidos es capaz de mantener indicadores de calidad de la atención que la validan como herramienta de atención clínica a distancia. La Telemedicina, en cuanto es una herramienta que acerca el especialista a comunidades alejadas de centros hospitalarios complejos, es valorada y muy bien calificada por los usuarios.

Palabras claves: Telemedicina, Tratamiento Anticoagulante, INR Capilar.

Correspondencia:

BQ. Ángela Roco Arriagada,

angela.roco@redsalud.gov.cl 


\section{Impact of tele-medicine for control of oral anticoagulant treatment}

Background: Starting in 2014 telemedicine has been used to control oral anticoagulant treatment (OAT) in patients attending a peripheral hospital (Curacavî), in connection with Hospital San Juan de Dios, based in Santiago.

Methods: A license for video conference was available to communicate both hospitals. Capillary INR and medications were available at Curacaví Hospital.

Results: Between June 2014 and December 2015, 2174 indications for OAT have been made through tele-medicine. Different estimates of quality of care and user satisfaction have been rated $\geq 6.7$ (1-7 sca1e). Percent of INR measurements in therapeutic range was $58.3 \%$ in Curacavi and 50.8\% at Hospital San Juan de Dios $(\mathrm{p}<0.05)$ and time in therapeutic range was $50.6 \%$ vs $42.6 \%$, respectively $(\mathrm{p}<0.05)$

Conclusion: Tele-medicine allowed a close relationship between remote medical facilities and a complex medical center and was fully validated as a means of controlling OAT with a high degree of acceptance by patients

Key words: Telemedicine, oral anticoagulant treatment, Capillary INR 
Introducción: La ampliación de las indicaciones de profilaxis antitrombótica con fármacos anti vitamina $\mathrm{K}$ en los últimos años y la longevidad de la población han incrementado de manera notable el número de enfermos susceptibles de beneficiarse de este tipo de tratamiento ${ }^{1-2}$. En Chile el anticoagulante más utilizado es el acenocumarol (en comprimidos de $4 \mathrm{mg}$ ), en los países anglosajones se utiliza la warfarina sódica y es con este medicamento con el que se han realizado la mayor parte de estudios y ensayos clínicos ${ }^{3}$. Los fármacos anti vitaminas $\mathrm{K}$ son eficaces, pero tienen un estrecho margen terapéutico y su eficacia se ve afectada por diferentes circunstancias como la alimentación o la administración de otros medicamentos ${ }^{4}$. Las principales consecuencias de los desajustes en las dosis son la trombosis (falta de eficacia) o el desarrollo de un evento hemorrágico por exceso de dosis. La minimización de estos riesgos exige controles periódicos para ajustar las dosis del fármaco a unos rangos de anticoagulación seguros, en función de una prueba analítica conocida como razón normalizada internacional $(\mathrm{INR})^{5}$. Existe una fuerte relación en entre el tiempo que los pacientes están en rango y la aparición de un efecto adverso, por lo que los controles del tratamiento con fármacos anti vitaminas $\mathrm{K}$ deben cumplir unos requisitos de calidad reconocidos internacionalmente ${ }^{1-2}$. Los principales son el porcentaje de pacientes en rango terapéutico y Tiempo en Rango Terapéutico (TTR) ${ }^{1-2-3}$. La masificación de las consultas del control del tratamiento con anti vitaminas $\mathrm{K}$, las dificultades de desplazamiento de los pacientes y la irrupción en el mercado de pequeños equipos que permiten la valoración del INR han motivado a las administraciones a iniciar programas de descentralización que van a afectar a un alto número de pacientes $^{6-7-8-9}$. Habitualmente, la totalidad de estas atenciones de salud son realizadas por médicos especialistas. No obstante, cada vez es más frecuente que los servicios de atención primaria colaboren para garantizar un mejor control del tratamiento anticoagulante oral así como facilitar al usuario la realización de las pruebas pertinentes ${ }^{2}$. El objetivo de este estudio fue implementar el control del tratamiento anticoagulante por Telemedicina entre el Hospital San Juan de Dios (alta complejidad) y el Hospital de Curacaví (baja complejidad) y evaluar su impacto en la calidad del tratamiento.

\section{Pacientes y métodos:}

En junio de 2014 se implementó un dispositivo de asistencia móvil en dependencias del Hospital de Curacaví y al mismo tiempo en el Hospital San Juan de Dios se instaló una licencia de videoconferencia utilizando la red MINSAL ubicada en el box de atención en el de Tratamiento Anticoagulante (TACO). Para la determinación de INR se instaló en la Unidad de Toma de Muestras del Hospital de Curacaví un equipo de Point of Care Testing (POCT) de INR Capilar (Coagucheck ${ }^{\circledR}$ Roche) conectado a un software de Tratamiento Anticoagulante (TAONet ${ }^{\circledR}$ Roche) ubicado en el computador del médico especialista del Hospital San Juan de Dios. Se dispuso un stock de Acenocumarol en la Farmacia del Hospital de Curacaví (no disponible en la canasta de fármacos de Atención Primaria), el cual se repone desde la Farmacia del Hospital San Juan de Dios vía receta.

El médico que realiza la interconsulta vía Telemedicina debe realizar un registro de las indicaciones generadas y enviarlas a través del software de dosificación de tratamiento anticoagulante TAONet ${ }^{\circledR}$, incluida la receta del fármaco anticoagulante para ser impresas en el Hospital de Curacaví. El equipo de este último, formado por un médico y dos enfermeros entrega la receta al paciente para que retire los fármacos en la Farmacia del Hospital de Curacaví. Una copia de la indicación es archivada en la ficha clínica del paciente. Tanto el médico como los enfermeros refuerzan las indicaciones entregadas por el médico especialista al paciente como a sus familiares. En la Figura 1 se indica el flujograma de la atención vía Telemedicina.

Análisis de datos: Los datos para el cálculo de Indicador Porcentaje INR dentro de rango y Tiempo en rango Terapéutico (TTR) se obtienen del software de dosificación de tratamiento anticoagulante TAONet ${ }^{\circledR}$. Para el análisis estadístico se utilizó test de chi2, mediante el programa estadístico Epiinfo ${ }^{7}$.

\section{Resultados}

En la Tabla 1 se indican las características de los pacientes del Hospital de Curacaví que se atendieron vía Telemedicina.

De junio 2014 a diciembre de 2015 se realizaron 2.174 consultas vía Telemedicina. La producción de Tele asistencias realizada por el Policlínico de TACO del SSMOC es la mayor de la Región Metropolitana (Figura 2).

Satisfacción Usuaria: Se constató una alta satisfacción usuaria al encuestar al los pacientes atendidos en Curacaví. Calificación igual o superiores a 6.8 (escala 1-7) se obtuvieron en los ítems calidad de la atención, trato de parte del personal, tiempo de espera en la toma de muestras y en la percepción de la calidad de la relación con el Servicio de Salud Occidente (Tabla 2) 
Figura 1: Flujograma de la atención por Telemedicina a paciente con tratamiento anticoagulante, HSJD y Hospital de Curacaví, SSMOC.
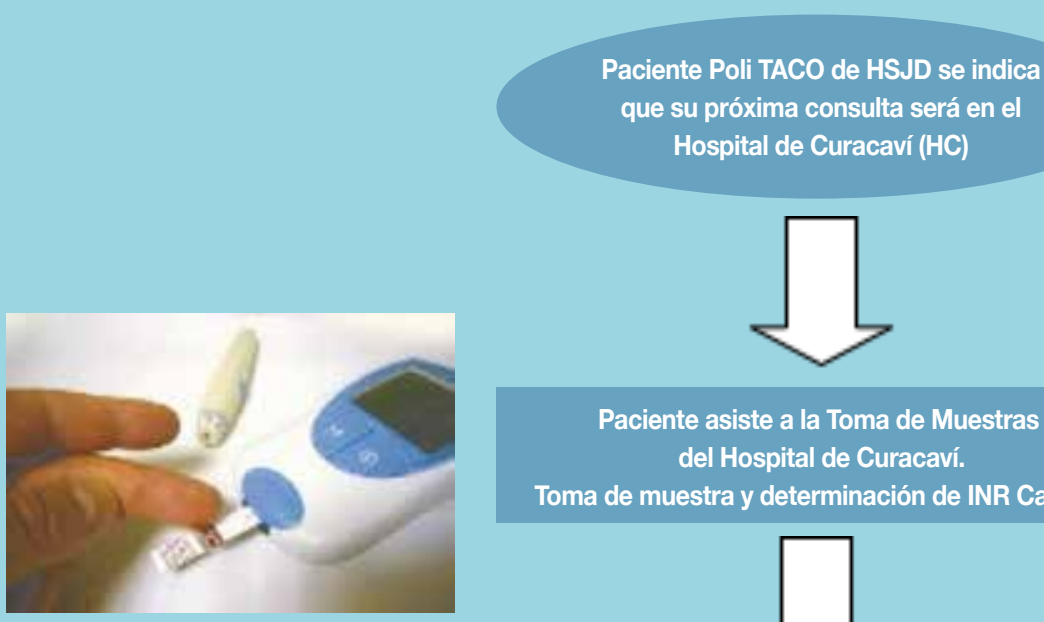

Toma de muestra y determinación de INR Capilar.

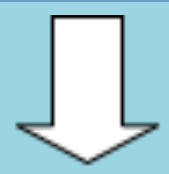

Se transmite el resultado del INR Capilar al software de TACO.

Médico Poli TACO en HSJD accede al resultado del examen.


Médico HSJD realiza consulta vía Telemedicina. Dosifica anticoagulante y agenda nuevo control.
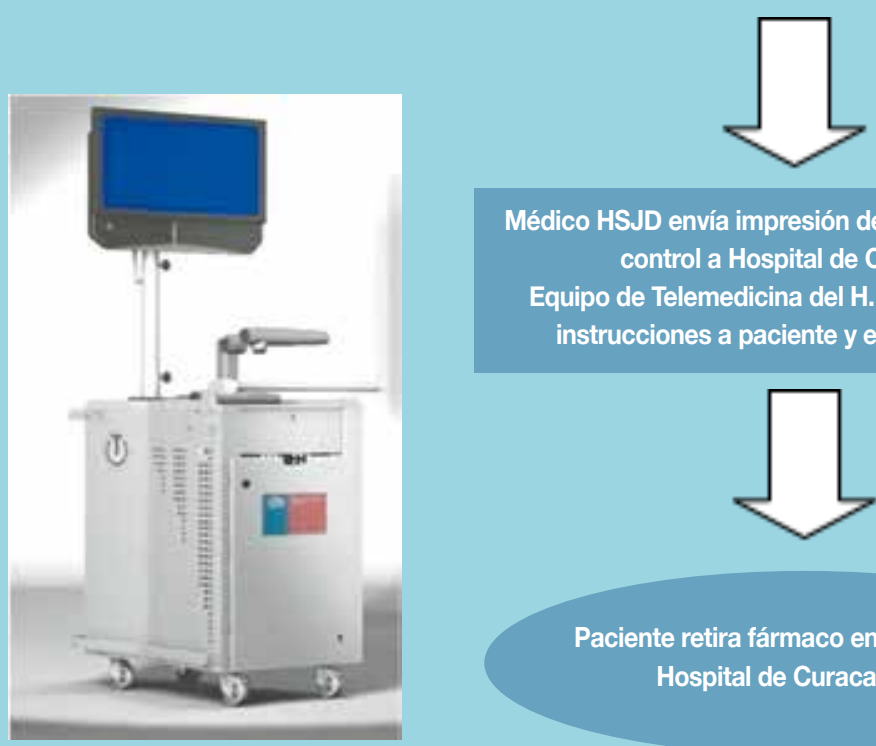

Médico HSJD envía impresión de receta y fecha de control a Hospital de Curacaví.

Equipo de Telemedicina del H. Curacaví reitera instrucciones a paciente y entrega receta.

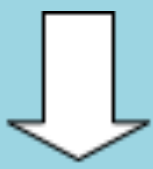

Paciente retira fármaco en Farmacia del Hospital de Curacaví (HC) 


\begin{tabular}{l|r}
\hline \multicolumn{2}{l}{ Tabla 1: Características de los pacientes de Poli TACO que se } \\
atendieron vía Telemedicina en Curacaví. \\
\hline Característica & $\mathbf{N}(\%)$ \\
\hline Hombres & $70(53,4 \%)$ \\
Mujeres & $61(45,5 \%)$ \\
Total & $131(100 \%)$ \\
Promedio Edad (años) \pm Desviación estándar & $69,23+/-15,54$ \\
\hline Diagnósticos & $\mathbf{N}(\%)$ \\
\hline Arritmia completa & $60(45,8 \%)$ \\
Trombosis & $25(19,1 \%)$ \\
Fibrilación & $11(8,4 \%)$ \\
Embolias (pulmonar o mesentérica) & $7(5,3 \%)$ \\
tt auricular & $5(3,8 \%)$ \\
Accidente Vascular encefálico & $4(3,1 \%)$ \\
Trombo (intra auricular/ intra ventricular) & $3(2,3 \%)$ \\
By pass extremidades & $2(1,5 \%)$ \\
Infarto cerebral & $2(1,5 \%)$ \\
Isquemia aguda extremidades & $2(1,5 \%)$ \\
Miocardiopatía dilatada & $2(1,5 \%)$ \\
Recambio valvular aórtico & $2(1,5 \%)$ \\
TIA & $2(1,5 \%)$ \\
Estenosis mitral & $1(0,8 \%)$ \\
Trombrome mitral & $1(0,8 \%)$ \\
& $1(0,8 \%)$ \\
\hline & $1(0,8 \%)$ \\
\hline
\end{tabular}

Figura 2: Producción Teleasistencias por Servicio de Salud región Metropolitana, Octubre 2012 a Abril 2015. (Fuente: Subsecretaria de Redes Asistenciales, Unidad Telemedicina)

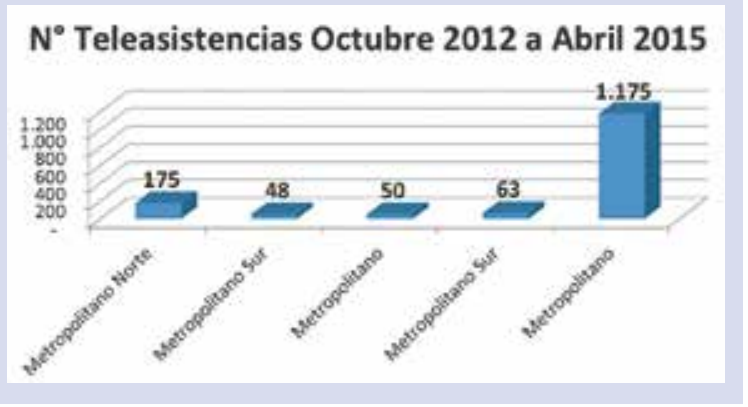

Indicadores de Calidad del Tratamiento: En el contexto del Monitoreo de indicadores de calidad del Tratamiento Anticoagulante, que también están incluidos en el proceso de Acreditación de Calidad de instituciones de salud (GCL 1.13, se han estandarizado dos Indicadores para la evaluación del TACO en toda la Red Occidente: 1) Porcentaje de determinaciones de INR en rango terapéutico; 2) Porcentaje de pacientes con INR dentro de rango terapéutico. Con la información de la base de datos y del módulo estadístico del software de tratamiento anti-
Tabla 2: Promedio notas por pregunta de encuesta de satisfacción usuaria realizada a pacientes Poli TACO Hospital de Curacaví posterior a implementación de INR Capilar y Telemedicina (Julio 2014).

\begin{tabular}{l|c|}
\hline Preguntas & Hospital de Curacaví \\
\hline $\begin{array}{l}\text { ¿Ha visto alguna mejora en la atención } \\
\text { recibida en la toma de muestras para } \\
\text { su control de Tratamiento Anticoagu- } \\
\text { lante en los últimos } 6 \text { meses? }\end{array}$ & 6,8 \\
\hline $\begin{array}{l}\text { ¿Cómo evalúa usted la atención por } \\
\text { parte del personal de Toma de Mues- } \\
\text { tras? }\end{array}$ & \multirow{2}{*}{6,9} \\
\hline $\begin{array}{l}\text { ¿Ha visto alguna mejora en la atención } \\
\text { en relación al tiempo de espera en la }\end{array}$ & \\
\hline $\begin{array}{l}\text { Toma de Muestras en los últimos } 6 \\
\text { meses? }\end{array}$ & 6,9 \\
\hline $\begin{array}{l}\text { Existe un buen trato en la atención que } \\
\text { usted recibe por parte de los profesio- } \\
\text { nales del establecimiento }\end{array}$ & \\
\hline $\begin{array}{l}\text { Cree usted que la red de atención del } \\
\text { Servicio de Salud Occidente se coordi- } \\
\text { na adecuadamente para brindarle una } \\
\text { atención correcta. }\end{array}$ & 6,9 \\
\hline
\end{tabular}

Tabla 3 A. INR dentro de rango terapéutico pacientes atendidos por Telemedicina en Hospital de Curacaví y pacientes atendidos en HSJD trimestralmente (umbral > $50 \%$ ) (Fuente: Software Tratamiento Anticoagulante, TAONet@). B. Tiempo en Rango Terapéutico pacientes atendidos por Telemedicina en Hospital de Curacaví y pacientes atendidos en HSJD trimestralmente (umbral > 60 \%). (Fuente: Software Tratamiento Anticoagulante, TAONet@).

\begin{tabular}{|c|c|c|c|c|c|}
\hline \multirow{2}{*}{ A } & \multicolumn{2}{|c|}{2014} & \multicolumn{3}{|c|}{2015} \\
\hline & $\begin{array}{l}\text { Julio a } \\
\text { Septiembre }\end{array}$ & $\begin{array}{l}\text { Octubre a } \\
\text { Diciembre }\end{array}$ & $\begin{array}{l}\text { Enero a } \\
\text { Marzo }\end{array}$ & $\begin{array}{l}\text { Abril a } \\
\text { Junio }\end{array}$ & $\begin{array}{l}\text { Julio a } \\
\text { Septiembre }\end{array}$ \\
\hline $\begin{array}{l}\text { Hospital San } \\
\text { Juan de Dios }\end{array}$ & $55,20 \%$ & $49,10 \%$ & $51,10 \%$ & $48,60 \%$ & $52,40 \%$ \\
\hline $\begin{array}{l}\text { Hospital de } \\
\text { Curacaví }\end{array}$ & $56,70 \%$ & $56,30 \%$ & $60 \%$ & $57,30 \%$ & $60,70 \%$ \\
\hline \multirow{2}{*}{ B } & \multicolumn{3}{|c|}{2014} & \multicolumn{2}{|l|}{2015} \\
\hline & $\begin{array}{l}\text { Julio a } \\
\text { Septiembre }\end{array}$ & $\begin{array}{l}\text { Octubre a } \\
\text { Diciembre }\end{array}$ & $\begin{array}{l}\text { Enero a } \\
\text { Marzo }\end{array}$ & $\begin{array}{l}\text { Abril a } \\
\text { Junio }\end{array}$ & $\begin{array}{l}\text { Julio a } \\
\text { Septiembre }\end{array}$ \\
\hline $\begin{array}{l}\text { Hospital San } \\
\text { Juan de Dios }\end{array}$ & $44,8 \%$ & $40,8 \%$ & $44,4 \%$ & $41,9 \%$ & $45,0 \%$ \\
\hline $\begin{array}{l}\text { Hospital de } \\
\text { Curacaví }\end{array}$ & $49,5 \%$ & $49,1 \%$ & $51,2 \%$ & $50,4 \%$ & $52,5 \%$ \\
\hline
\end{tabular}


coagulante fue posible conocer el porcentaje (\%) de INR dentro del rango terapéutico en un período de tiempo determinado. Este indicador se midió trimestralmente en la Tabla 3A se muestra un comparativo entre los pacientes atendidos por Telemedicina y el equipo local del Hospital de Curacaví y los pacientes atendidos por el mismo médico especialista en el Hospital San Juan de Dios. Respecto del Tiempo en Rango Terapéutico (TTR), que mide el tiempo cronológico durante el cual el paciente se encuentra dentro del rango de INR asignado a su patología, se incluyen los pacientes que reciben anticoagulante oral para rango de INR de 2,0 a 3,0 portadores de cualquier tipo de trastorno del ritmo. Los resultados de TTR para pacientes del Hospital de Curacaví y Hospital San Juan de Dios se muestran en Tabla 3B.

\section{Discusión}

La producción de tele asistencias realizada por el Policlínico de TACO del SSMOC es la mayor de toda la Región Metropolitana (Figura 2).La implementación de INR Capilar y consulta vía Telemedicina ha sido muy bien recibida por los usuarios quienes califican con un promedio de 6,9 (escala 1-7) esta estrategia (Tabla 2).

El impacto en la calidad del tratamiento ha sido evidente, lo que se observa en los indicadores tanto de porcentaje INR en rango terapéutico como para el TTR, los cuales fueron mayores en el $\mathrm{H}$. Curacaví que en el Hospital San Juan de Dios. (58.3\% vs 50.8\%, respectivamente (p $<0,05)$. En el caso de TTR 50,6\% de los pacientes del Hospital de Curacaví se encuentran en rango versus un 46,2\% de los pacientes del Hospital San Juan de Dios $(p<0,05)$. Probablemente estos resultados se deban al reforzamiento de las indicaciones entregadas en el control por el médico especialista por el equipo de Telemedicina local tanto al paciente como a sus familiares, así como a la mejora en la adherencia a la terapia -pilar de la calidad de la misma- derivada de la facilitación en el acceso al control percibido por los pacientes. Lo anterior ha significado menor riesgo de trombosis o hemorragia en estos pacientes, evitando de esta manera consultas al Servicio de Urgencia y hospitalizaciones (datos preliminares no publicados).

En 2005, la Organización Mundial de la Salud (OMS) estableció un observatorio global para la salud de e-health. Este supervisa el desarrollo de tecnologías de información y comunicaciones (ICT) para la asistencia médica, incluyendo Telemedicina, y permite proporcionar información confiable para elaborar mejores prácticas, políticas y normas ${ }^{11-12}$. Hasta el momento, la mayor parte de las experiencias en Telemedicina han ocurrido en países industrializados y aún hay poca experiencia en los países en desarrollo. La ventaja fundamental de este recurso tecnológico se centra en mejorar el acceso, deviniendo en una herramienta útil para reducir inequidades y reforzar sistemas de salud en países en vía de desarrollo ${ }^{13-14}$. Este es el primer estudio en Chile que evalúa el impacto de la Telemedicina en la Calidad del tratamiento Anticoagulante.

\section{Conclusiones}

Lecciones aprendidas: Para conseguir resultados, un servicio de Telemedicina debe formar parte de una red colaborativa, cubrir las necesidades reales de los profesionales de salud locales, utilizar tecnologías simples y tener al menos algunos componentes cara a cara. Si se aplica a los problemas sanitarios para los que existe una alta y creciente demanda de asistencia, como es el tratamiento Anticoagulante oral, este tipo de servicio puede ser económicamente viable y ayudar a mejorar el acceso del paciente a la asistencia de salud especializada en todas las Regiones en Chile. La Telemedicina utilizada por equipos comprometidos, es capaz de mantener indicadores de calidad de la atención que la validan como herramienta de atención clínica a distancia. La Telemedicina, en cuanto es una herramienta que acerca el especialista a comunidades alejadas de centros hospitalarios complejos, es aceptada, valorada y muy bien calificada por los usuarios. 


\section{Referencias}

1. NAVARRO J, CESARA J, FERNÁNDEZ M, FONTCUBERTA J, REVERTER J, GOL-FREIXA J. Morbilidad y mortalidad en pacientes con tratamiento anticoagulante oral, Rev Esp Cardiol. 2007; 60:1226-32.

2. DURÁN C, RODRÍGUEZ C, TATO F, ALONSO N, LADO F. Anticoagulación oral, AN. MED. INTERNA 2003; 20: 377-384

3. MARÍN A, NEIRA V, AIZMAN A, PAREDES A, PALMA $\mathrm{S}, \mathrm{RUIZ} \mathrm{M}$, et al. Eficacia y seguridad del tratamiento anticoagulante oral con antagonistas de vitamina $\mathrm{K}$ en pacientes con prótesis valvulares cardíacas, Rev Chil Cardiol 2014; 33 : 27-32.

4. ANSELL J, HIRSH J, POLLER L, BUSSEY H, JACOBSON A, HYLEK E. The pharmacology and management of the vitamin K antagonist: The seventh ACCP conference on antithrombotic and thrombolytic therapy. Chest. 2004; 126:S234-64.

5. HIRSH J, POLLER L, PATH FRC. The International Normalized Ratio: A Guide to Understanding and Correcting Its Problems, Arch Intern Med, 1997, 154: 282-288

6. VAN DEN BESSELAAR A, PÉQUÉRIAUX N, EBBEN M, VAN DER FEEST J, DE JONG K, GANZEBOOM M, et al. Point-of-care monitoring of vitamin K-antagonists: validation of CoaguChek XS test strips with International Standard thromboplastin, J Clin Pathol 2012; 65:1031-1035.

7. BRIGGS C. Guidelines for point-of-care testing: haematology, British Journal of Haematology, 2008; 142: 904-915.

8. Medical Advisory Secretariat. Point-of-care international normalized ratio (INR) monitoring devices for patients on longterm oral anticoagulation therapy: an evidence-based analysis.
Ontario Health Technology Assessment Series 2009; 9.

9. BUBNER T, LAURENCE C, GIALAMAS A, YELLAND L, RYAN P, WILLSON K, et al. Effectiveness of point-of-care testing for therapeutic control of chronic conditions: results from the PoCT in General Practice Trial, MJA 2009; 190: 624-626.

10. Manual del Estándar General de Acreditación para Prestadores Institucionales de Atención Cerrada, disponible en: http://www. supersalud.gob.cl/568/articles-4530_Manual_AC_pdf.pdf

11. Telemedicine - opportunities and developments in Member States [Internet]. Geneva: World Health Organization; 2011. Disponible en: http://whqlibdoc.who.int/publications/2010/9789241564144_eng.pdf

12. WOOTTON R, GEISSBUHLER A, JETHWANI K, KOVARIK C, PERSON D, VLADZYMYRSKYY A, ZANABONIA $\mathrm{P}$, ZOLFOG M, Long-running telemedicine networks delivering humanitarian services: experience, performance and scientific output, Bull World Health Organ 2012;90:341-347D

13. ALKMIM, M., MINELLI FIGUEIRA, R, SORIANO MARCOLINO, M., SILVA CARDOSO, C., PENA DE ABREU,M., RODRIGUES CUNHA,L., FERREIRA DA CUNHA, D., PIRES ANTUNES, A., DE A RESENDE ,A., SANTOS RESENDEG, E., PINHO RIBEIROH, A., Improving patient access to specialized health care: the Telehealth $\mathrm{Ne}$ twork of Minas Gerais, Brazil, Bull World Health Organ 2012;90:373-378.

14. WOOTTON R, PATIL NG, SCOTT RE, HO K, editors. Telehealth in the developing world. London: RSM Press; 2009. Disponible en: http://www.idrc.ca/EN/Resources/Publications/ Pages/IDRCBookDetails.aspx?PublicationID=57 